\title{
Association between the rate of fluoroquinolones- resistant Gram-negative bacteria and antibiotic consumption from China based on 145 tertiary hospitals data in 2014
}

\section{Ping Yang \\ Zhejiang University \\ Yunbo Chen \\ Zhejiang University \\ Saiping Jiang \\ Zhejiang University \\ Ping Shen \\ Zhejiang University \\ Xiaoyang Lu \\ Zhejiang University}

Yonghong Xiao ( $\nabla$ xiaoyonghong@zju.edu )

Zhejiang University School of Medicine First Affiliated Hospital https://orcid.org/0000-0003-2529-938X

\section{Research}

Keywords: Fluoroquinolones-resistant; Escherichia coli; Klebsiella pneumoniae; Pseudomonas aeruginosa; Acinetobacter baumannii; Antibiotic consumption.

Posted Date: November 15th, 2019

DOI: https://doi.org/10.21203/rs.2.17294/v1

License: (9) This work is licensed under a Creative Commons Attribution 4.0 International License.

Read Full License

Version of Record: A version of this preprint was published at BMC Infectious Diseases on April 7th, 2020. See the published version at https://doi.org/10.1186/s12879-020-04981-0. 


\section{Abstract}

This study aimed to investigate the relationship between the rate of fluoroquinolones-resistant (FQR) gram-negative bacteria and antibiotic consumption intensity in 145 tertiary hospitals from China in 2014. Methods A retrospective study using national surveillance data from 2014 was conducted. Data on the annual consumption of each antibiotic, and the rate of FQR gram-negative bacteria, were collected from each participating hospital, and the correlation between antibiotic consumption and FQR rate was simultaneously investigated.Results The overall antibiotic consumption intensity among the hospitals varied between 23.93 and 115.39 defined daily dosages (DDDs) per 100 patient-days (median, 46.30 DDDs per 100 patient-days). Cephalosporins were the most commonly prescribed antibiotics, followed by fluoroquinolones, penicillins, and carbapenems, and the rate of FQR gram-negative bacteria from each hospital varied. The correlation analysis showed significantly relationship between the percentage of FQR E. coli and the consumption of FQs consumption $(r=0.308, p<0.01)$ and levofloxacin $(r=0.252, p<0.01)$. For FQR K. pneumoniae, not only FQs $(r=0.291, p<0.01)$ and levofloxacin $(r=0.260, p<0.01)$ use but also carbapenems $(r=0.242, p<0.01)$ and overall antibiotics $(r=0.247, p<0.01)$ use showed significant correlation. A strong correlation was observed between the resistant proportion of FQR P. aeruginosa and the consumption of all antibiotics $(r=0.260, p<0.01), F Q s(r=0.319, p<0.01)$ and levofloxacin $(r=0.377$, $p<0.01)$. The percentage of levofloxacin-resistant $A$. baumannii was significantly correlated with the consumption of all antibiotics $(r=0.282, p<0.01)$, third-generation cephalosporins excluding combinations with beta-lactamase inhibitors $(r=0.246, p<0.01), F Q s(r=0.254, p<0.01)$ and levofloxacin $(r=0.336$, $p<0.01)$. However, the correlation of the ciprofloxacin-resistant $A$. baumannii and the antibiotics consumption was not found.Conclusions A significant relationship was demonstrated between the antibiotic consumption and the rates of FQR gram-negative bacteria. As unreasonable antibiotics usage remains crucial in the proceeding of resistant bacteria selection, our study could greatly promote the avoidance of unnecessary antibiotic usage.

\section{Background}

Fluoroquinolones (FQs) were introduced as broad-spectrum antibiotics. Because of their excellent oral bioavailability, FQs have been widely prescribed to patients with bacterial infections. With the widespread use of FQs, FQs-resistant (FQR) gram-negative bacteria are gradually increasing, limiting the choices of antibiotics available for treating infections.

According to surveillance report of China in the first half of 2018, the overall prevalence of ciprofloxacinresistant strains was $57.8 \%$ in Escherichia coli, $35.4 \%$ in Klebsiella spp., $17.1 \%$ in P. aeruginosa and $75.4 \%$ in $A$. baumannii respectively. FQR gram-negative bacteria isolates were reported as independent risk factors for in-hospital mortality, which have been associated with greater hospital expenses and poor clinical outcomes ${ }^{1-5}$. Moreover, compared with other phenotypic resistance patterns, FQR E. coli and Klebsiella spp hospital-onset bacteremia had a larger relative impact on mortality ${ }^{6}$. It is reported that previous colonization of FQR E. coli can lead to the spread of extended-spectrum beta-lactamase (ESBL) after the use of quinolone prophylaxis ${ }^{5,7}$. The high probability of ESBL production by FQR gram-negative 
bacteria makes anti-infective treatment more difficult. The risk of gram-negative bloodstream infection increased over time in parallel with an increased FQR rate, and the unadjusted mortality rate was $18 \%$ for FQR gram-negative bloodstream infections ${ }^{1,8}$.

It is plausible that the irrational use of antibiotics can increase selective pressure of bacterial resistance, which is considered to be the main factor contributing to the emergence of resistance. In this study, we investigated the correlation between the FQR rate of gram-negative bacteria and antibiotic consumption.

\section{Methods}

\subsection{Study design}

A cross-sectional study including 145 voluntarily participating hospitals was conducted. Data on antibiotic consumption and the FQR rate of gram-negative bacteria from inpatients at each participating hospital in 2014 were collected. Then the correlation between antibiotic consumption and resistant rate was performed.

\subsection{Data collection}

Each participating hospital reported annual data of 2014. They were required to report antibiotic consumption data to national antibacterial drug clinical consumption survey network. While bacterial resistance data were obtained from China antimicrobial resistance surveillance system.

Administrative data of each participating hospital, including hospital type, administrative region, number of beds, admissions and patient-days was recorded,.

\subsection{Measurement of Antibiotic Consumption}

Hospital pharmacists reported each antibiotic consumption to the national antibacterial drug clinical consumption survey network annually. According to Anatomic Therapeutic Chemical (ATC) classification system $^{9}$, data on consumption of all antibiotics (J01), beta-lactams (J01C+J01D), beta-lactam-betalactamase inhibitor combinations, beta-lactams excluding combinations with beta-lactamase inhibitors (CBLI), penicillins (J01C), penicillins excluding CBLI (J01C-J01CR), cephalosporins (J01DB+J01DC+J01DD+J01DE), cephalosporins excluding CBLI, third-generation cephalosporins (J01DD), third-generation cephalosporins excluding CBLI, fourth-generation cephalosporins (J01DE), carbapenems (J01DH), FQs (J01MA), ciprofloxacin, levofloxacin and moxifloxacin were analyzed. Antibiotic consumption was expressed as antibiotics consumption intensity, which was indicated as the number of defined daily dose (DDDs) per 100 patient-days. According to the World Health Organization ATC/DDD classification, DDD is the assumed average maintenance dose per day for a drug used for its main indication in adults. Patient-days were defined as number of discharged patients during the same period multiplied by the average length of stay of hospitalized patients in the same period. Then, data on antibiotic consumption were analyzed, according to the main classes. 


\subsection{Antibiotic resistance}

Each participating hospital was required to report data about E. coli, K. pneumoniae, P. aeruginosa and $A$. baumannii, isolated from all sample sources (e.g., bloodstream, respiratory tract, urinary tract, wound pus, other sterile body fluids, cerebrospinal fluid, genital tract and others). The duplicate strains were excluded, isolates of each species from each patient who recovered within 7 days were considered duplicate. Then, the number of isolates that were FQR and the total number of bacterial strain isolated from clinical specimens were collected. All hospitals must adhered to the Clinical and Laboratory Standards Institute 2014 guidelines and antibiotic susceptibility tests are performed with a routine laboratory method. FQsresistance was defined as a strain resistant to levofloxacin or ciprofloxacin. If the resistance rates were different, the higher resistance rate was considered as the resistance rate of the strain to FQs. The FQR rate was calculated as the number of $\mathrm{FQR}$ isolates divided by the total number of the isolates of the same species tested multiplied by 100 . For $A$. baumannii, ciprofloxacin and levofloxacin-resistant rate were derived separately because of their great differences. Each laboratory can ensure the consistency and standardized assessment of the antimicrobial resistance data through quality control. The antibiotic resistance data were processed by Whonet 5.6 software. Quality control bacterial strains were $E$. coli ATCC25922, K. pneumoniae ATCC700603, P. aeruginosa ATCC27853 and A. baumannii ATCC19606. The hospital with isolated strains less than 50 isolates per year was excluded from the study.

\subsection{Statistical analysis}

With pearson's correlation analysis, the association between annual antibiotic consumption and FQR rate of gram-negative bacteria from 145 hospitals of China was performed. The relationship between the FQR rate of gram-negative bacteria and the consumption intensity of overall antibiotics, beta-lactams, betalactam-beta-lactamase inhibitor combinations, beta-lactams excluding CBLI, penicillins, penicillins excluding CBLI, cephalosporins, cephalosporins excluding CBLI, third-generation cephalosporins, thirdgeneration cephalosporins excluding CBLI, fourth-generation cephalosporins, carbapenems, FQs, ciprofloxacin, levofloxacin and moxifloxacin were analyzed individually. Statistical significance was defined as $p<0.05$. All the analyses were performed using Microsoft Excel 2013 and STATA 20.0 (StataCorp LLC, Texas, USA).

\section{Results}

\subsection{Participating hospitals}

A total of 145 hospitals were included in this study. 141 of the participating hospitals were tertiary hospitals, which are the highest quality hospitals in China. There are 29 hospitals in North China, 29 hospitals in East China, 22 hospitals in Central China, 25 hospitals in Southern China, 12 hospitals in the southwest of China, 15 hospitals in the northwest of China, and 13 hospitals in northeast of China. These hospitals had a median of 2356 beds (range, $720-8475$ beds). The median number of inpatients per year reached 88.3 thousands (range, 10-410 thousands). 


\subsection{Antibiotics consumption}

During the study period, the overall antibiotics consumption intensity for the participating hospitals varied from 23.93 to 115.39 DDDs/100 patient-days, the median was 46.30 DDDs/100 patient-days. Among them, cephalosporins were the most commonly prescribed antibiotics, followed by fluoroquinolone, penicillins and carbapenems. The antibiotics consumption intensity for the main antibiotic classes was depicted in Table 1.

\subsection{Correlation between antibiotics consumption intensity and FQR E. coli}

138 hospitals were included to perform the correlation between antibiotics consumption intensity and rate of FQR E. coli. 158866 strains of $E$. coli were isolated, and 94247 strains of them were FQR. The percentage of $E$. coli isolates that were resistant to FQs at each hospital was 44.96-82.2\%, and the median value was $61.67 \%$. The percentage of FQR E. coli was significantly positively correlated with the

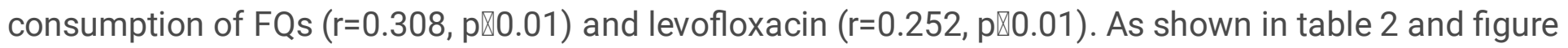
1.

\section{Table 1. Antibiotics consumption intensity for the main classes of antibiotics in $\mathbf{1 4 5}$ hospitals}

Class (ATC category)

\begin{tabular}{|c|c|}
\hline & 100 patient-days) \\
\hline All antibiotics $\square 01 \square$ & $46.30(23.93-115.39)$ \\
\hline Beta-lactams $\square 01 \mathrm{C}+\mathrm{J} 01 \mathrm{D} \square$ & $33.03(19.35-67.05)$ \\
\hline Beta-lactam-beta-lactamase inhibitor & $7.34(0.73-34.33)$ \\
\hline \multicolumn{2}{|l|}{ combinations } \\
\hline Beta-lactams excluding CBLI & $25.95(13.48-58.82)$ \\
\hline Penicillins $\square 01 \mathrm{C} \square$ & $5.69(0.99-23.21)$ \\
\hline Penicillins excluding CBLI & $2.40(0.02-21.89)$ \\
\hline 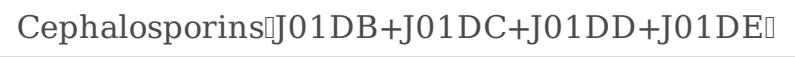 & $24.70(10.81-52.52)$ \\
\hline Cephalosporins excluding CBLI & $19.82(9.20-51.38)$ \\
\hline 3-GC口J01DD! & $10.99(2.57-38.98)$ \\
\hline 3GC excluding CBLI & $5.99(1.05-20.99)$ \\
\hline 4-GC口J01DE凸 & $0.30(0-5.98)$ \\
\hline Carbapenems $\square 01 \mathrm{DH} \square$ & $1.95(0.17-10.06)$ \\
\hline Fluoroquinolones $\square 01 \mathrm{MA} \square$ & $5.70(1.58-19.25)$ \\
\hline Ciprofloxacin (J01MA02) & $0.09(0-2.80)$ \\
\hline Levofloxacin (J01MA12) & $3.73(0.01-13.56)$ \\
\hline Moxifloxacin (J01MA14) & $1.41(0-6.74)$ \\
\hline
\end{tabular}


ATC: Anatomic Therapeutic Chemical; DDDs: defined daily dosages; CBLI: combinations with beta-lactamase inhibitors; 3-GC: the third generation cephalosporins; 4-GC: the fourth generation cephalosporins.

\subsection{Correlation between antibiotics consumption intensity and FQR K. pneumoniae}

139 hospitals were included to analyze the correlation between antibiotic consumption intensity and rate of FQR K. pneumoniae. 194957 strains of K. pneumoniae were isolated, and 48287 strains of them were FQR. The percentage of $K$. pneumoniae isolates that were resistant to FQs at each hospital was 5.3$66.9 \%$, and the median value was $23.1 \%$. The percentage of FQR K. pneumoniae was significantly correlated with the consumption of all antibiotics $(r=0.247, p \otimes 0.01)$, carbapenems $(r=0.242, p \otimes 0.01), F Q$ $(r=0.291, p \otimes 0.01)$ and levofloxacin $(r=0.260, p \otimes 0.01)$. As demonstrated in table 2 and figure 2.

\subsection{Correlation between antibiotics consumption intensity and FQR $P$. aeruginosa}

139 hospitals were included to analyze the correlation between antibiotic consumption intensity and rate of FQR $P$. aeruginosa. 111711 strains of $P$. aeruginosa were isolated, and 27171 strains of them were FQR. The percentage of $P$. aeruginosa isolates that were resistant to FQs at each hospital was 7.7-65.2\%, and the median value was $22.4 \%$. The percentage of FQR $P$. aeruginosa was significantly correlated with the consumption of all antibiotics $(r=0.260, p \otimes 0.01), F Q s(r=0.319, p \otimes 0.01)$ and levofloxacin $(r=0.377, p \rrbracket$ 0.01). As demonstrated in table 2 and figure 3.

\subsection{Correlation between antibiotics consumption intensity and FQR A. baumannii}

131 hospitals were included to analyze the correlation between antibiotic consumption intensity and rate of levofloxacin-resistant A. baumannii. 93114 strains of $A$. baumannii were isolated, and 52695 strains of them were levofloxacin-resistant. The percentage of $A$. baumannii isolates that were resistant to levofloxacin at each hospital was $16.1-93.9 \%$, and the median value was $59.3 \%$. The percentage of levofloxacin-resistant $A$. baumannii was significantly correlated with the consumption of all antibiotics $(r=0.282, p \otimes 0.01)$, third-generation cephalosporins excluding CBLI $(r=0.246, p \otimes 0.01), F Q s(r=0.254, p \otimes 0.01)$

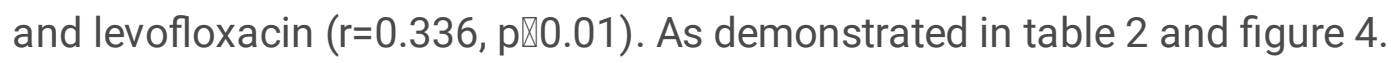

133 hospitals were included to analyze the correlation between antibiotic consumption intensity and rate of ciprofloxacin-resistant $A$. baumannii. 101374 strains of $A$. baumannii were isolated, and 80032 strains of them were ciprofloxacin-resistant. The percentage of $A$. baumannii isolates that were resistant to ciprofloxacin at each hospital was $28.7-95 \%$, and the median value was $77.6 \%$. The percentage of ciprofloxacin-resistant $A$. baumannii was not significantly correlated with the antibiotics consumption. As demonstrated in table 2. 
Table 2 Correlations between main classes of antibiotics consumption intensity and the rate of fluoroquinolone-resistant gram-negative bacteria 


\begin{tabular}{|c|c|c|c|c|c|c|c|c|c|c|}
\hline & \multicolumn{2}{|c|}{$\begin{array}{l}\text { fluoroquinolone- } \\
\text { resistant }\end{array}$} & \multicolumn{2}{|c|}{$\begin{array}{l}\text { fluoroquinolone- } \\
\text { resistant }\end{array}$} & \multicolumn{2}{|c|}{$\begin{array}{l}\text { fluoroquinolone- } \\
\text { resistant }\end{array}$} & \multicolumn{2}{|c|}{$\begin{array}{l}\text { Levofloxacin- } \\
\text { resistant }\end{array}$} & \multicolumn{2}{|c|}{$\begin{array}{l}\text { Ciprofloxacin- } \\
\text { resistant }\end{array}$} \\
\hline & \multicolumn{2}{|c|}{ E. coli } & \multicolumn{2}{|c|}{ K. pneumoniae } & \multicolumn{2}{|c|}{ P. aeruginosa } & \multicolumn{2}{|c|}{ A. baumannii } & \multicolumn{2}{|c|}{ A. baumannii } \\
\hline & \multicolumn{2}{|c|}{$n=138$} & \multicolumn{2}{|c|}{$n=139$} & \multicolumn{2}{|c|}{$\mathrm{n}=139$} & \multicolumn{2}{|c|}{$\mathrm{n}=131$} & \multicolumn{2}{|c|}{$n=133$} \\
\hline & $r^{a}$ & $p^{*}$ & $r$ & $p$ & $r$ & $p$ & $r$ & $p$ & $r$ & $p$ \\
\hline All antibiotics $\square 01 \square$ & 0.174 & 0.062 & $0.247 *$ & 0.003 & $0.260 *$ & 0.002 & $0.282 *$ & 0.001 & 0.194 & 0.055 \\
\hline Beta-lactams $\square 01 C+J 01 D \square$ & -0.035 & 0.682 & 0.116 & 0.173 & 0.109 & 0.202 & 0.165 & 0.060 & 0.165 & 0.058 \\
\hline $\begin{array}{l}\text { Beta-lactam-beta-lactamase inhibitor } \\
\text { combinations }\end{array}$ & -0.176 & 0.093 & 0.135 & 0.113 & -0.046 & 0.589 & -0.039 & 0.656 & 0.037 & 0.671 \\
\hline Beta-lactams excluding CBLI & 0.118 & 0.167 & 0.088 & 0.303 & 0.152 & 0.075 & 0.200 & 0.072 & 0.154 & 0.077 \\
\hline Penicillins $\square 01 \mathrm{C} \square$ & -0.118 & 0.169 & 0.040 & 0.643 & 0.126 & 0.140 & 0.081 & 0.359 & 0.036 & 0.678 \\
\hline Penicillins excluding CBLI & 0.004 & 0.964 & 0.046 & 0.590 & 0.212 & 0.072 & 0.215 & 0.064 & 0.078 & 0.371 \\
\hline Cephalosporins $\square$ J01DB+J01DC+J01DD+J01DE $\square$ & -0.014 & 0.873 & 0.068 & 0.426 & 0.061 & 0.478 & 0.157 & 0.074 & 0.185 & 0.053 \\
\hline Cephalosporins excluding CBLI & 0.095 & 0.269 & 0.034 & 0.693 & 0.091 & 0.286 & 0.139 & 0.112 & 0.141 & 0.106 \\
\hline 3-GC $\square J 01 D D \square$ & -0.055 & 0.524 & 0.147 & 0.085 & 0.063 & 0.459 & 0.194 & 0.067 & 0.156 & 0.073 \\
\hline 3GC excluding CBLI & 0.093 & 0.276 & 0.131 & 0.125 & 0.109 & 0.203 & $0.246^{*}$ & 0.005 & 0.133 & 0.127 \\
\hline 4-GC口J01DE $\square$ & 0.037 & 0.671 & -0.063 & 0.460 & 0.044 & 0.611 & -0.182 & 0.077 & -0.028 & 0.748 \\
\hline Carbapenems $\square 01 \mathrm{DH} \square$ & 0.129 & 0.131 & $0.242 *$ & 0.004 & -0.065 & 0.445 & -0.003 & 0.975 & 0.002 & 0.983 \\
\hline Fluoroquinolones $\square \mathrm{J} 01 \mathrm{MA} \square$ & $0.308^{* *}$ & 0.000 & $0.291 *$ & 0.001 & $0.319 *$ & 0.000 & $0.254^{*}$ & 0.003 & 0.159 & 0.068 \\
\hline Ciprofloxacin (J01MA02) & -0.014 & 0.869 & 0.075 & 0.383 & 0.054 & 0.532 & -0.021 & 0.811 & 0.077 & 0.376 \\
\hline Levofloxacin (J01MA12) & $0.252^{*}$ & 0.003 & $0.260^{*}$ & 0.002 & $0.377^{*}$ & 0.000 & $0.336^{*}$ & 0.000 & 0.157 & 0.072 \\
\hline
\end{tabular}


ATC: Anatomic Therapeutic Chemical; CBLI: combinations with beta-lactamase inhibitors; 3-GC: the third generation cephalosporins; 4-GC: the fourth generation cephalosporins.

\section{Discussion}

The present study reflects the current status of antibiotic usage and antimicrobial resistance patterns at the hospital level in China. During the study period, cephalosporins were found to be the most commonly prescribed antibiotics, followed by fluoroquinolone, penicillins, and carbapenems. The percentage of FQR gram-negative bacteria from each hospital varied. Our data demonstrated that the percentage of $F Q R$. coli was significantly positively correlated with the consumption of FQs and levofloxacin while the rate of FQR K. pneumoniae was significantly correlated with the consumption of all antibiotics, carbapenems, FQs and levofloxacin. When it comes to FQR P. aeruginosa, the resistant rate was significantly correlated with the consumption of all antibiotics, FQs and levofloxacin. Furthermore, the percentage of levofloxacin-resistant $A$. baumannii was significantly correlated with the consumption of all antibiotics, third-generation cephalosporins excluding combinations with beta-lactamase inhibitors, FQs and levofloxacin. However, the correlation of ciprofloxacin-resistant $A$. baumannii and the antibiotics consumption was not found.

In accordance with previous studies, our results found that the FQR E. coli was significantly associated with FQs consumption ${ }^{9-18}$. The most important resistance mechanism of $E$. coli to quinolones is mutation of target gene DNA gyrase and topoisomerase IV. Further, up expression of active efflux pump, changes in membrane permeability of bacteria and plasmid-mediated quinolone resistance are the main mechanism of FQR E. coli production ${ }^{19}$. However, the emergence of FQR E. coli occurs as a multistep process, with increasing numbers of target gene mutations leading to progressively higher minimum inhibitory concentrations ${ }^{20}$. Not surprisingly, the most important risk factor for FQR E. coli appears to be previous FQs use ${ }^{21-23}$. Restriction of quinolone use can offer opportunities to reduce the prevalence of FQR E. coli ${ }^{24-26}$.

However, data from 42 Spanish hospitals collected by the European Antimicrobial Surveillance Network indicate that amoxicillin clavulanic acid use is the main driving force for the progression of FQR E. coli, possibly due to its high consumption in Spain ${ }^{12} .36$ acute care hospitals from France indicated the level of first, second and third-generation cephalosporins, as well as tetracycline's usage influenced the incidence of FQR E. coli ${ }^{14}$. As $E$. coli are less often resistant to third-generation cephalosporins than to quinolones, infections due to FQR E. coli may be treated with third-generation cephalosporins. Hence, the use of third-generation cephalosporins might be higher because the incidence of FQR E. coli has increased. The author believe that the reason for the correlation between FQR E. coli and tegacycline use is that FQR E. coli is plausibly associated with resistance to tetracyclines. 
There is mounting evidence demonstrating that the prevalence of ciprofloxacin-resistant K. pneumoniae was associated with use of ciprofloxacin and FQs ${ }^{17,26,27}$. A study from database of the Korean Health Insurance Review and Assessment Service suggested that the consumption of all third-generation cephalosporins was significantly correlated with resistance rates of $K$. pneumoniae to levofloxacin with a quarter $\operatorname{lag}^{28}$. The resistance mechanism of $K$. pneumoniae to FQs is the change of target sites, the change of outer membrane protein permeability, the effect of efflux pump and the transfer of resistant plasmids among bacteria. Our study results suggested that the percentage of FQR K. pneumoniae was significantly correlated with the consumption of all antibiotics, carbapenems, FQs and levofloxacin. The use of FQs is a risk factor for the incidence of FQR K. pneumoniae ${ }^{29}$. Therefore, it is no doubt that the overuse of FQs increases FQR K. pneumoniae.

The drug resistance of $K$. pneumoniae is mostly mediated by plasmids. Plasmid DNA can carry multiple drug-resistant genes such as ESBLs, Amp C enzyme and metalloenzyme-coded genes at the same time. As a mobile genetic primitive, plasmids can transmit carbapenemase-producing resistance genes, resulting in the outbreaks of multidrug-resistant $K$. pneumoniae ${ }^{30}$. Therefore, it is credible that the production of multidrug-resistant $K$. pneumoniae was correlated with the use of carbapenems and all antibiotics.

Resistance to fluoroquinolones developed in $P$. aeruginosa by various mechanisms: mutations in the genes encoding bacterial DNA topoisomerase II and topoisomerase IV is a major cause of resistance to fluoroquinolones in $P$. aeruginosa isolates. Meanwhile, overexpression of active efflux systems can reduce the permeability of the membrane. Furthermore, the plasmid carries the gene of beta-lactamase, which leads to the high level of resistance to quinolones in ESBL-producing strains ${ }^{31}$.

Consistent with our study, the positive correlation between FQs consumption and the rates of FQR $P$. aeruginosa was described ${ }^{17,26,32-34}$. Moreover, the increased consumption of levofloxacin but not ciprofloxacin was associated with an increased incidence of FQR $P$. aeruginosa ${ }^{35-39}$. The differential effects of ciprofloxacin and levofloxacin on the risk of isolating FQR $P$. aeruginosa might be related to the greater intrinsic in vitro activity of ciprofloxacin against $P$. aeruginosa than that of levofloxacin. With higher minimum inhibitory concentration against $P$. aeruginosa, levofloxacin might have been more likely than ciprofloxacin to select for colonization or infection with FQR P. aeruginosa. However, various studies indicated that the consumption of ceftazidime, anti-pseudomonal cephalosporin and ciprofloxacin was positively correlated with the incidence rates of ciprofloxacin-resistant $P$. aeruginosa ${ }^{34,37,40}$. As we known, the use of certain antibiotics could be both a cause and a consequence of the resistance emergence. So it seems reasonable to explain higher rates of consumption of ceftazidime, anti-pseudomonal cephalosporin and ciprofloxacin in hospitals is likely to be a result of the high prevalence of FQR organisms and the use of alternative drugs to treat infection with these organisms.

A. baumannii has become a difficult bacteria in clinical treatment because of its complex drug resistance mechanism and high drug resistance rate. The use of widespectrum antibiotics will further screen out multidrug-resistant bacteria. Our study showed that the resistance rate of $A$. baumannii to ciprofloxacin 
was higher than that of levofloxacin, probably because ciprofloxacin is easier to detect the overexpression of pumps ${ }^{41}$. Our study illustrated the selection pressure of FQs use in the development of FQR A. baumannii, which is in accordance with a nationwide multicenter study from Korea ${ }^{17}$. The drug resistance mechanism of $A$. baumannii to quinolones modified the bacterial DNA helix enzyme through the mutation of quinolone resistance gene cluster, thus reducing the affinity between the drug and the enzyme-DNA complex and leading to drug resistance. Also, some efflux systems affect the drug sensitivity. Up to now, three types of plasmid-mediated quinolone resistance genes have been identified, namely aminoglycoside acetyltransferase AAC (6') - Ib-cr, specific efflux systems Qep A and Oqx AB and Qnr family ${ }^{42}$. Furthermore, simultaneous mutations in gyrA and parC genes are expected to play a major role in high-level fluoroquinolone resistance in A. baumannif ${ }^{43}$.

Amp $\mathrm{C}$ enzyme is a cephalosporin enzyme encoded by chromosomes inherent in all $A$. baumannii. Adding the promoter insertion sequence ISAba1 beside the Amp C gene increases the production of betalactamase, which leads to resistance to cephalosporins. This also explains the correlation between drug resistance and consumption of $3 \mathrm{GC}$ excluding $\mathrm{CBLI}{ }^{44}$. A domestic research report that the consumption of carbapenem has a significant positive relationship with $A$. baumannii resistance to levofloxacin ${ }^{45}$. A retrospective study manifested the consumption of cefmetazole and total cephamycin positively correlated with the resistance rates of $A$. baumannii to levofloxacin. The author believed that these consequences may be partly due to production of AmpC enzymes ${ }^{46}$.

With the extensive use of FQs, their adverse reactions and the harm caused by irrational use have become increasingly prominent in recent years. Therefore, drug regulatory agencies at home and abroad frequently issued drug safety warnings, demanding the discontinuation or restriction of the FQs use. In our survey, the resistant rates of all gram-negative bacteria were significantly correlated with the FQs consumption, which is consistant with previous study. It is more likely that the resistance mechanism of gram-negative bacteria to FQs leads to a correlation between resistance and FQs consumption. Therefore, in view of the adverse reactions of FQs and the increasing drug resistance, controlling FQs consumption should be taken seriously.

Antibiotic use is a major risk factor in development of antibiotic resistance but the relationship is complex with additional factors involved such as cross-transmission, interhospital transfer of resistance, a community contribution to resistance, and a complex relationship between resistance and the use of a variety of antibiotics. In our study, the relationships between FQs use and resistance to gram-negative bacteria represent the hospital-level perspective, not that of the patient. Therefore, the results may be subject to ecological bias, meaning that findings may not reflect patient-level relationships. Additionally, lack of data on outpatient use of antibiotics could have led to an underestimation of the magnitude of association between FQs exposure and the subsequent isolation of resistant bacteria. It would be great to know the patterns of the resistant isolates. If so, we can conclude definitely whether any outbreak or clonal spread influenced our results. If clonal dissemination of resistant strains were responsible for some portion of the resistant organisms present, then we might expect to find a weaker association 
between antibiotic exposure and resistance. While we acknowledge that our research did not take chronology into account, which is an inevitable defect of the design. This may also be the reason for the not very high correlation coefficient. Even so, it must be stated that the scale of the study made it the most comprehensive investigation reported domestically.

\section{Conclusion}

To summarize, in this study we quantified the associations between antibiotic consumption and the incidence of FQR gram-negative bacteria. As prudent and responsible use of antibiotics remains crucial in preventing the selection of resistant bacteria, our study results could serve as a driving force for implementation of antimicrobial stewardship policies.

\section{Abbreviations}

A.baumannii: Acinetobacter baumannii; ATC: Anatomic Therapeutic Chemical; DDDs: Defined daily dosages; E. coli: Escherichia coli; ESBLs: Extended-spectrum beta-lactamase; FQs: Fluoroquinolones; FQR: fluoroquinolones-resistant; K. pneumoniae: Klebsiella pneumoniae, P. aeruginosa: Pseudomonas aeruginosa.

\section{Declarations}

\section{Ethics approval and consent to participate}

Given that this study was performed without accessing patient information, approval of the ethics committee was not required

\section{Consent for publication}

Not applicable

\section{Availability of data and material}

The data used in the current study are available from the corresponding author on reasonable request.

\section{Competing interests}

The authors report no conflicts of interest relevant to this article.

\section{Funding}

This study was sponsored by National Natural Science Foundation of China (81361138021, 81711530049), Key Research and Development Program of Zhejiang Province (2015C03032).

\section{Authors' contributions}


Ping Yang conducted the correlation analysis and prepared the initial drafts of the manuscript. Yunbo Chen and Ping Shen were responsible for the bacterial resistance data analysis. Saiping Jiang contributed in antibiotic consumption analysis. Xiaoyang Lu was responsible for the results interpretation and manuscript review. Yonghong Xiao as a principal investigator designed the study, collected the data and revised the manuscript. All authors contributed to the final version of the manuscript. All authors read and approved the final manuscript.

\section{Acknowledgements}

Not applicable

\section{References}

1. Kadri SS, Adjemian J, Lai YL, et al. Difficult-to-Treat Resistance in Gram-negative Bacteremia at 173 US Hospitals: Retrospective Cohort Analysis of Prevalence, Predictors, and Outcome of Resistance to All First-line Agents. Clin Infect Dis. 2018 67(12):1803-1814.

2. Camins BC, Marschall J, DeVader SR, et al. The clinical impact of fluoroquinolone resistance in patients with E coli bacteremia. J Hosp Med. 2011;6(6):344-349.

3. Lautenbach E, Metlay JP, Bilker WB, et al. Association between fluoroquinolone resistance and mortality in Escherichia coli and Klebsiella pneumoniae infections: the role of inadequate empirical antimicrobial therapy. Clin Infect Dis. 2005;41(7):923-929.

4. Gasink LB, Fishman NO, Weiner MG, et al. Fluoroquinolone-resistant Pseudomonas aeruginosa: assessment of risk factors and clinical impact. Am J Med. 2006;119(6):526.e519-525.

5. Sawa T, Shimizu M, Moriyama K, et al. Association between Pseudomonas aeruginosa type III secretion, antibiotic resistance, and clinical outcome: a review. Crit Care. 2014 18(6):668.

6. Suzuki H, Perencevich EN, Livorsi DJ, et al. Attributable mortality due to fluoroquinolone and extended-spectrum cephalosporin resistance in hospital-onset Escherichia coli and Klebsiella spp bacteremia: A matched cohort study in 129 Veterans Health Administration medical centers. Infect Control Hosp Epidemiol. 2019;40(8):928-931.

7. Chong Y, Shimoda S, Yakushiji H, et al. Clinical impact of fluoroquinolone-resistant Escherichia coli in the fecal flora of hematological patients with neutropenia and levofloxacin prophylaxis. PLoS One. 2014;9(1):e85210.

8. Blennow $\mathrm{O}$, Ljungman P, Sparrelid E, et al. Incidence, risk factors, and outcome of bloodstream infections during the pre-engraftment phase in 521 allogeneic hematopoietic stem cell transplantations. Transpl Infect Dis. 2014;16(1):106-114.

9. McDonnell L, Armstrong D, Ashworth M, et al. National disparities in the relationship between antimicrobial resistance and antimicrobial consumption in Europe: an observational study in 29 countries-authors' response. J Antimicrob Chemother. 2017;72(12):3500. 
10. Gallini A, Degris E, Desplas M, et al. Influence of fluoroquinolone consumption in inpatients and outpatients on ciprofloxacin-resistant Escherichia coli in a university hospital. J Antimicrob Chemother. 2010;65(12):2650-2657.

11. Hsu LY, Tan TY, Tam VH, et al. Surveillance and correlation of antibiotic prescription and resistance of Gram-negative bacteria in Singaporean hospitals. Antimicrob Agents Chemother. 2010;54(3):11731178.

12. Cuevas 0 , Oteo J, Lázaro E, et al. Significant ecological impact on the progression of fluoroquinolone resistance in Escherichia coli with increased community use of moxifloxacin, levofloxacin and amoxicillin/clavulanic acid. J Antimicrob Chemother. 2011;66(3):664-669.

13. Wu HH, Liu HY, Lin YC, et al. Correlation between levofloxacin consumption and the incidence of nosocomial infections due to fluoroquinolone-resistant Escherichia coli. J Microbiol Immunol Infect. 2016;49(3):424-429.

14. Batard E, Ollivier F, Boutoille D, et al. Relationship between hospital antibiotic use and quinolone resistance in Escherichia coli. Int J Infect Dis. 2013;17(4):e254-258.

15. Hsueh PR, Chen WH, Luh KT. Relationships between antimicrobial use and antimicrobial resistance in Gram-negative bacteria causing nosocomial infections from 1991-2003 at a university hospital in Taiwan. Int J Antimicrob Agents. 2005;26(6):463-472.

16. Kim YA, Park YS, Youk T, et al. Trends in South Korean antimicrobial use and association with changes in Escherichia coli resistance rates: 12-year ecological study using a nationwide surveillance and antimicrobial prescription database. PLoS One. 2018;13(12):e0209580.

17. Kim B, Kim Y, Hwang H, et al. Trends and correlation between antibiotic usage and resistance pattern among hospitalized patients at university hospitals in Korea, 2004 to 2012: A nationwide multicenter study. Medicine (Baltimore). 2018;97(51):e13719.

18. Cusini A, Herren D, Bütikofer L, et al. Intra-hospital differences in antibiotic use correlate with antimicrobial resistance rate in Escherichia coli and Klebsiella pneumoniae: a retrospective observational study. Antimicrob Resist Infect Control. 2018;7(89).

19. Redgrave LS, Sutton SB, Webber MA, et al. Fluoroquinolone resistance: mechanisms, impact on bacteria, and role in evolutionary success. Trends Microbiol. 2014;22(8):438-445.

20. Singh R, Swick MC, Ledesma KR, et al. Temporal interplay between efflux pumps and target mutations in development of antibiotic resistance in Escherichia coli. Antimicrob Agents Chemother. 2012;56(4):1680-1685.

21. Bedoin M, Cazorla C, Lucht F, et al. Risk factors for quinolone-resistance in women presenting with Escherichia coli acute pyelonephritis. Med Mal Infect. 2014;44(5):206-216.

22. Kim J, Kang Cl, Joo EJ, et al. Risk factor of community-onset spontaneous bacterial peritonitis caused by fluoroquinolone-resistant Escherichia coli in patients with cirrhosis. Liver Int. 2014;34(5):695-699.

23. Katsandri A, Avlamis A, Vasilakopoulou A, et al. Risk factors for coexistence of fluoroquinolone resistance and ESBL production among Enterobacteriaceae in a Greek university hospital. J 
Chemother. 2008;20(4):452-457.

24. Gottesman BS, Carmeli Y, Shitrit P, et al. Impact of quinolone restriction on resistance patterns of Escherichia coli isolated from urine by culture in a community setting. Clin Infect Dis. 2009;49(6):869-875.

25. Sarma JB, Marshall B, Cleeve V, et al. Effects of fluoroquinolone restriction (from 2007 to 2012) on resistance in Enterobacteriaceae: interrupted time-series analysis. J Hosp Infect. 2015;91(1):68-73.

26. Wang $\mathrm{H}$, Wang $\mathrm{H}$, Yu X, et al. Impact of antimicrobial stewardship managed by clinical pharmacists on antibiotic use and drug resistance in a Chinese hospital, 2010-2016: a retrospective observational study. BMJ Open. 2019;9(8):e026072.

27. Lee HS, Loh YX, Lee JJ, et al. Antimicrobial consumption and resistance in five Gram-negative bacterial species in a hospital from 2003 to 2011. J Microbiol Immunol Infect. 2015;48(6):647-654.

28. Ryu S, Klein EY, Chun BC. Temporal association between antibiotic use and resistance in Klebsiella pneumoniae at a tertiary care hospital. Antimicrob Resist Infect Control. 2018;7:83.

29. Bolon MK, Wright SB, Gold HS, et al. The magnitude of the association between fluoroquinolone use and quinolone-resistant Escherichia coli and Klebsiella pneumoniae may be lower than previously reported. Antimicrob Agents Chemother. 2004;48(6):1934-1940.

30. Navon-Venezia S, Kondratyeva K, Carattoli A. Klebsiella pneumoniae: a major worldwide source and shuttle for antibiotic resistance. FEMS Microbiol Rev. 2017;41(3):252-275.

31. Subedi D, Vijay AK, Willcox M. Overview of mechanisms of antibiotic resistance in Pseudomonas aeruginosa: an ocular perspective. Clin Exp Optom. 2018 Mar;101(2):162-171. 2018.

32. Lafaurie M, Porcher R, Donay JL, et al. Reduction of fluoroquinolone use is associated with a decrease in methicillin-resistant Staphylococcus aureus and fluoroquinolone-resistant Pseudomonas aeruginosa isolation rates: a 10 year study. J Antimicrob Chemother 2012;67(4):1010-1015.

33. Weng TC, Chen YH, Lee CC, et al. Correlation between fluoroquinolone consumption in hospitals and ciprofloxacin resistance amongst Pseudomonas aeruginosa isolates causing healthcare-associated infections, Taiwan, 2000-2009. Int J Antimicrob Agents. 2011;37(6):581-584.

34. Rogues AM, Dumartin C, Amadéo B, et al. Relationship between rates of antimicrobial consumption and the incidence of antimicrobial resistance in Staphylococcus aureus and Pseudomonas aeruginosa isolates from 47 French hospitals. Infect Control Hosp Epidemiol. 2007;28(12):13891395.

35. Lee YJ, Liu HY, Lin YC, et al. Fluoroquinolone resistance of Pseudomonas aeruginosa isolates causing nosocomial infection is correlated with levofloxacin but not ciprofloxacin use. Int $J$ Antimicrob Agents. 2010;35(3):261-264.

36. Kaye KS, Kanafani ZA, Dodds AE, et al. Differential effects of levofloxacin and ciprofloxacin on the risk for isolation of quinolone-resistant Pseudomonas aeruginosa. Antimicrob Agents Chemother. 2006;50(6):2192-2196.

37. Mohr JF, Jones A, Ostrosky-Zeichner L, et al. Associations between antibiotic use and changes in susceptibility patterns of Pseudomonas aeruginosa in a private, university-affiliated teaching 
hospital: an 8-year-experience: 1995-2002. Int J Antimicrob Agents. 2004 Oct;24(4):346-51.

38. Pakyz AL, Lee JA, Ababneh MA, et al. Fluoroquinolone use and fluoroquinolone-resistant Pseudomonas aeruginosa is declining in US academic medical centre hospitals. J Antimicrob Chemother. 2012;67(6):1562-1564.

39. Polk RE, Johnson CK, McClish D, et al. Predicting hospital rates of fluoroquinolone-resistant Pseudomonas aeruginosa from fluoroquinolone use in US hospitals and their surrounding communities. Clin Infect Dis. 2004;39(4):497-503.

40. Gbaguidi-Haore H, Dumartin C, L'Hériteau F, et al. Antibiotics involved in the occurrence of antibioticresistant bacteria: a nationwide multilevel study suggests differences within antibiotic classes. $J$ Antimicrob Chemother. 2013;68(2):461-470.

41. Güler G, Eraç B. Investigation of fluoroquinolone resistance mechanisms in clinical Acinetobacter baumannii isolates. Mikrobiyol Bul. 2016;50(2):278-286.

42. Asif M, Alvi IA, Rehman SU. Insight into Acinetobacter baumannii: pathogenesis, global resistance, mechanisms of resistance, treatment options, and alternative modalities. Infect Drug Resist. . 2018;11:1249-1260.

43. Ardebili A, Lari AR, Beheshti M, et al. Association between mutations in gyrA and parC genes of Acinetobacter baumannii clinical isolates and ciprofloxacin resistance. Iran J Basic Med Sci. 2015;18(6):623-626.

44. Hamidian M, Hall RM. Resistance to third-generation cephalosporins in Acinetobacter baumannii due to horizontal transfer of a chromosomal segment containing ISAba1-ampC. J Antimicrob Chemother. 2014;69(10):2865-2866.

45. Cao J, Song W, Gu B, et al. Correlation between carbapenem consumption and antimicrobial resistance rates of Acinetobacter baumannii in a university-affiliated hospital in China. J Clin Pharmacol. 2013 53(1):96-102.

46. Song W, Cao J, Mei YL. Correlation between cephamycin consumption and the incidence of antimicrobial resistance in Acinetobacter baumannii at a university hospital in China from 2001 to 2009. Int J Clin Pharmacol Ther. 2011;49(12):765-771.

\section{Figures}




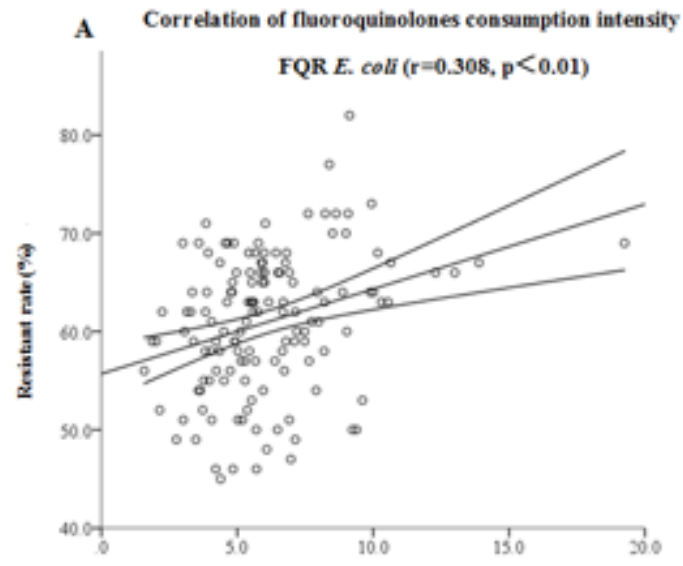

Fluoroquinolones consumption intensity (DDDs per 100 patient-days)

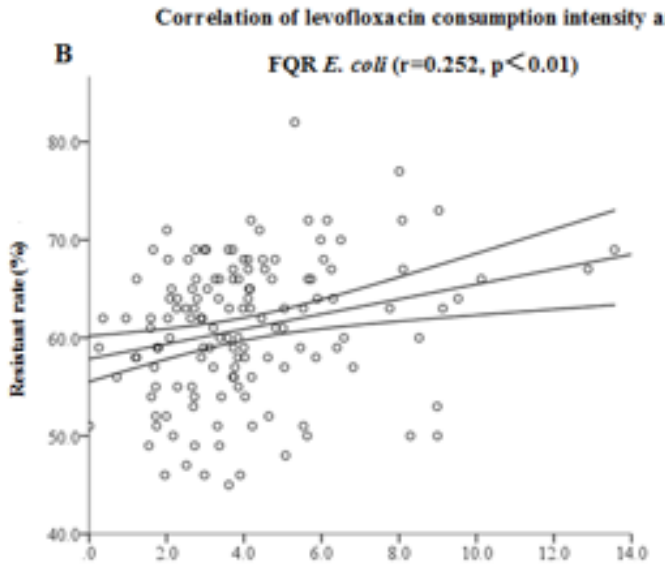

Levofnoxacin consumption intensiry (DDDs per 100 patieat-days)

\section{Figure 1}

Correlation of FQR E. coli and consumption intensity of (A) fluoroquinolones; (B) levofloxacin.

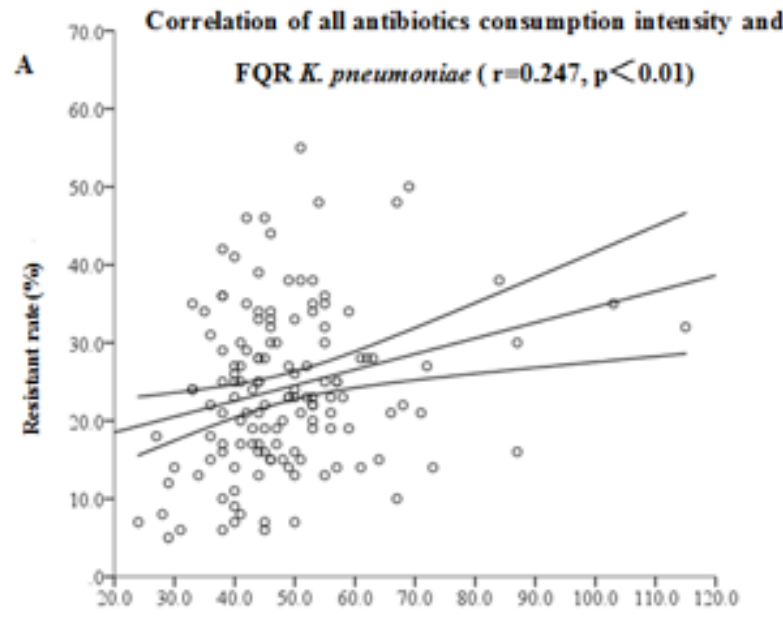

All antibiotics consumption intensity (DDDs per 100 patient-days)

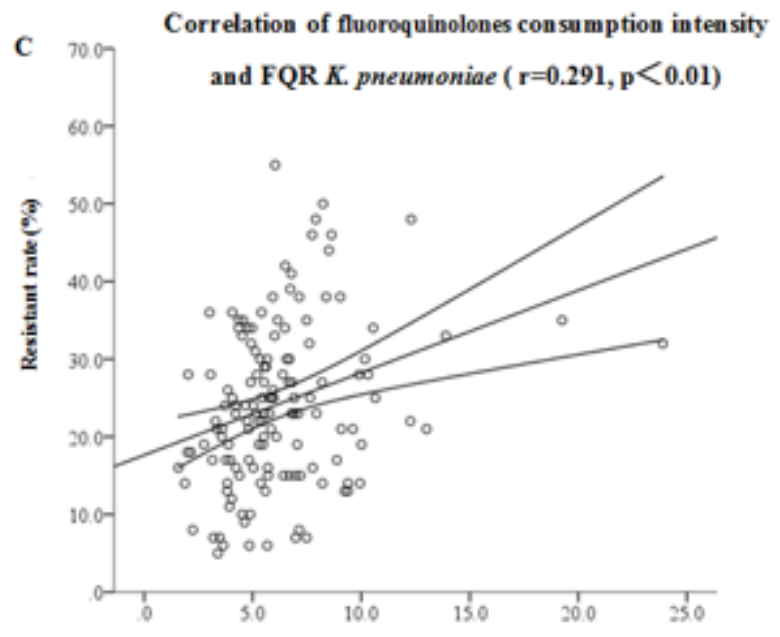

Fluoroquinolones consump tion intensity (DDDs per 100 patient-days)

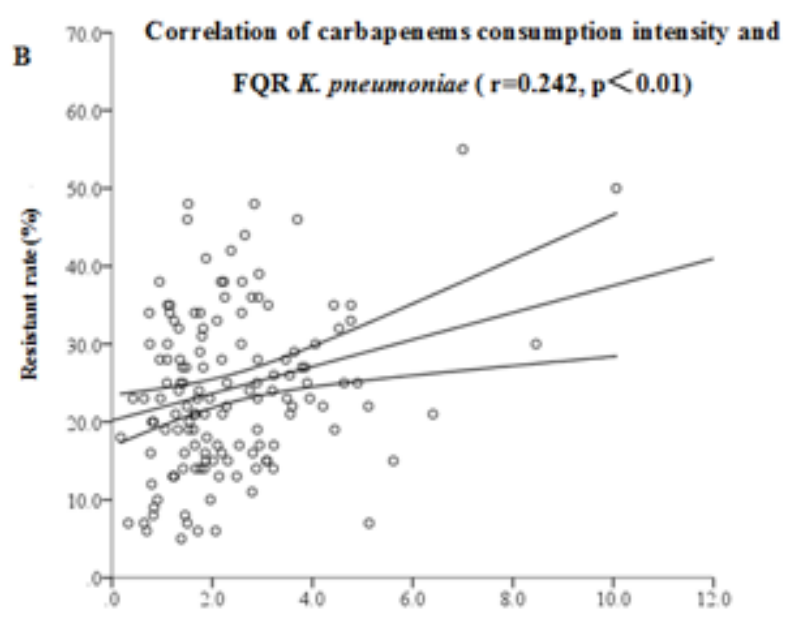

Carbapenems consump tion intensity (DDDs per 100 patient-days)

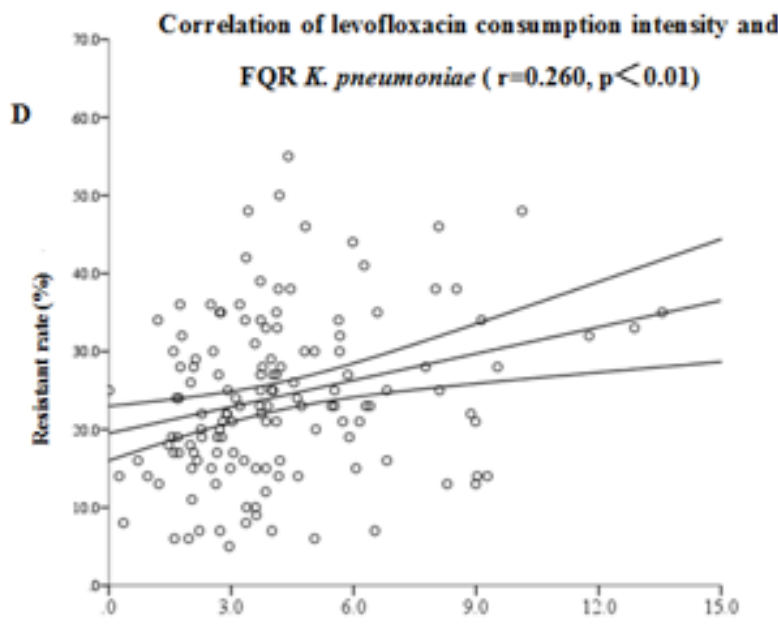

Levofloxacin consumption intensity (DDDs per 100 patient-days) 
Figure 2

Correlation of FQR K. pneumoniae and consumption intensity of (A) all antibiotics; (B) carbapenems; (C) fluoroquinolones; (D) levofloxacin.

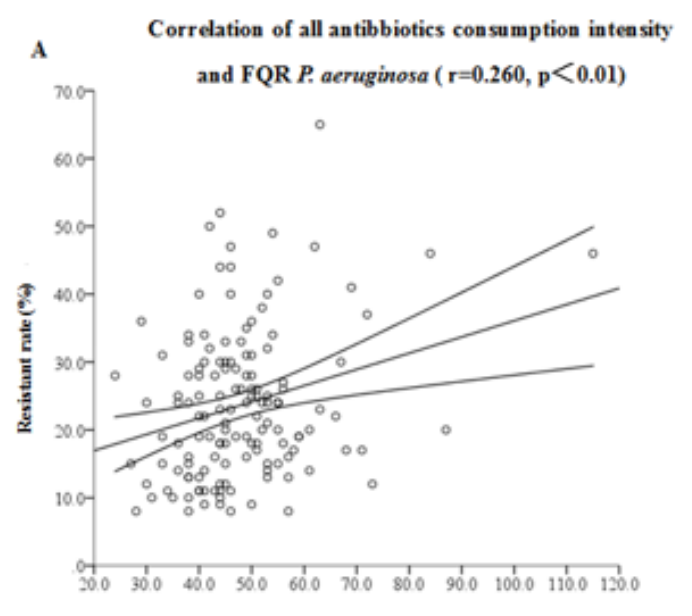

All antibiotics consump tion intensity (DDDs per 100 patient-days)

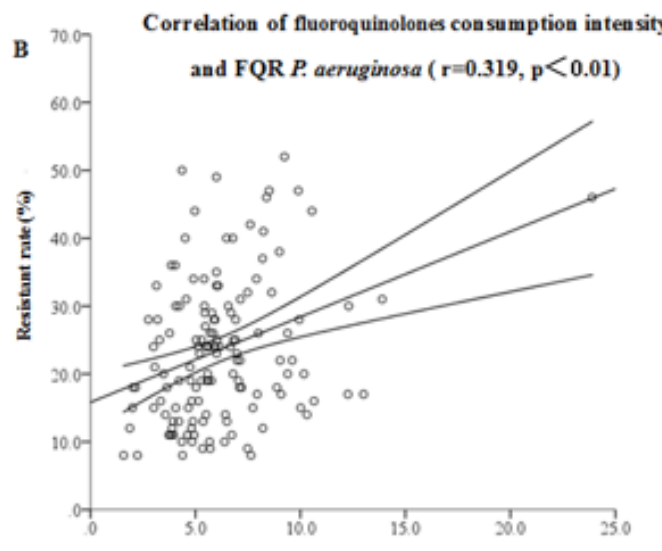

Fluoroquinolones consump tion intensity (DDDs per 100 patient-days)

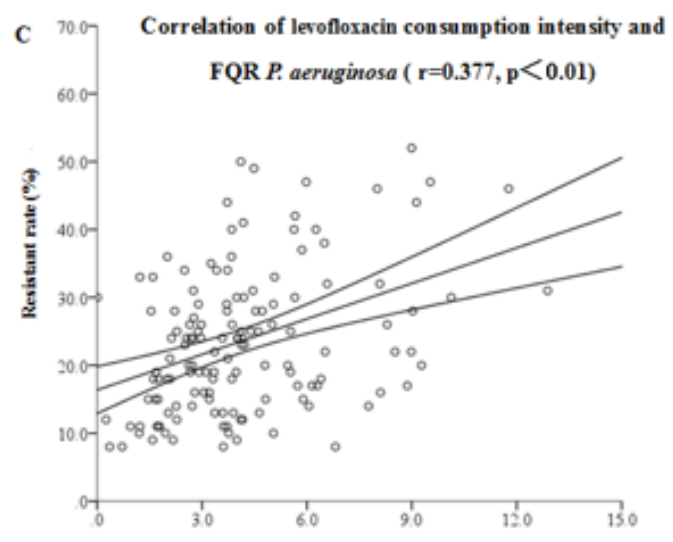

Levofloxacin consumption intensity (DDDs per 100 patient-days)

\section{Figure 3}


Correlation of FQR P. aeruginosa and consumption intensity of (A) all antibiotics; (B) fluoroquinolones; (C) levofloxacin.

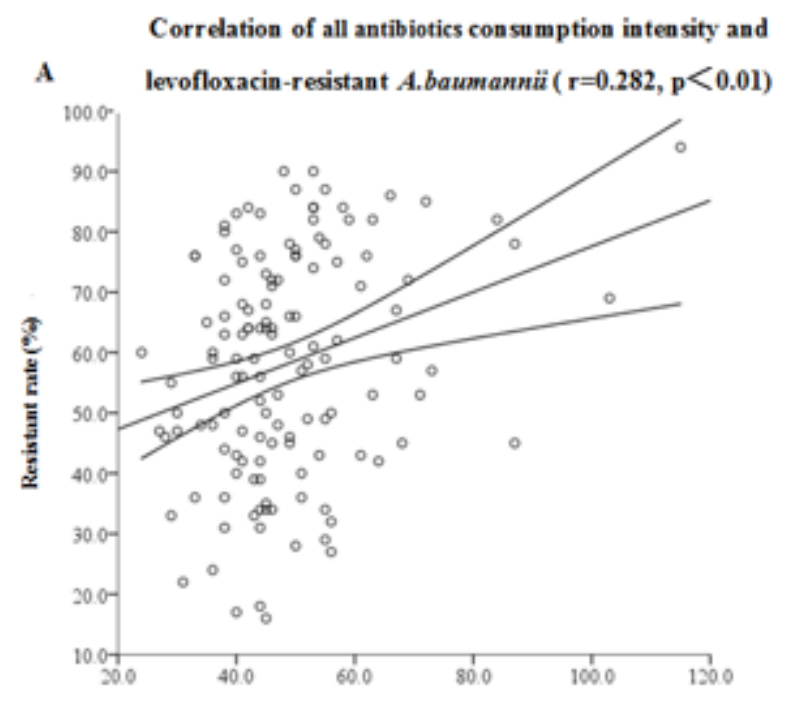

All antibiotics consump tion intensity (DDDs per 100 patient-days)

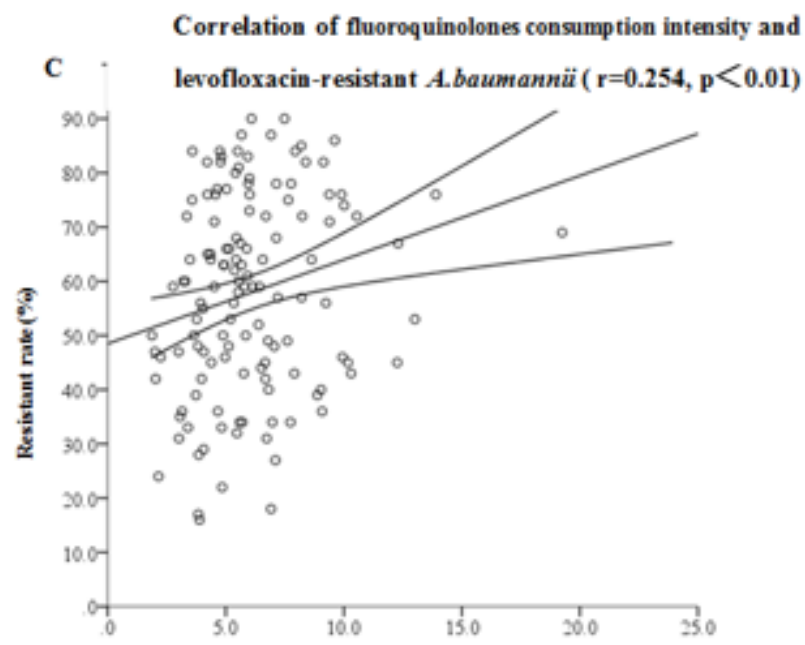

Fluoroquinolones consump tion intensity (DDDs per 100 patient-days)

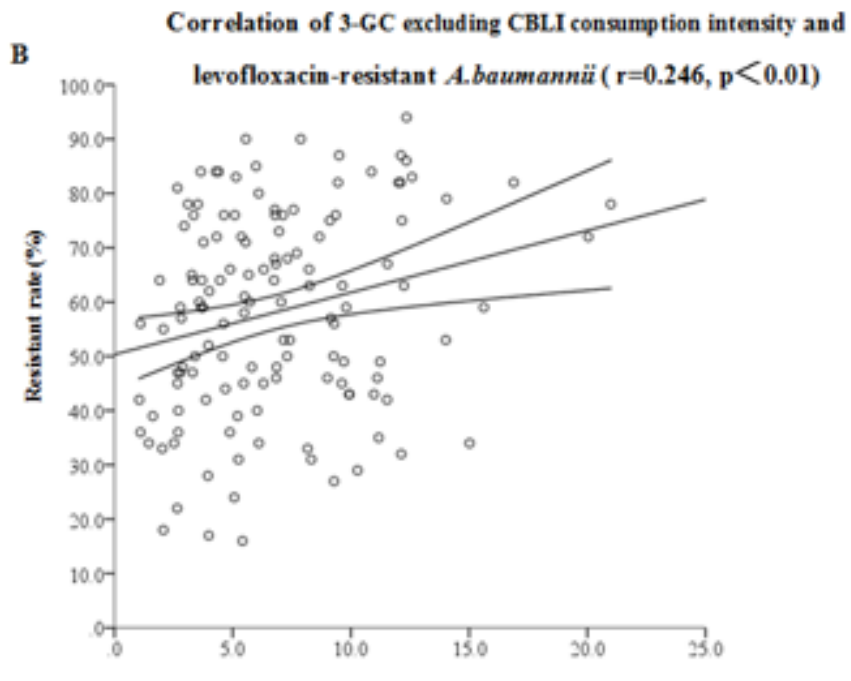

3-GC excluding CBLI consump tion intensity (DDDs per 100 patient-days)

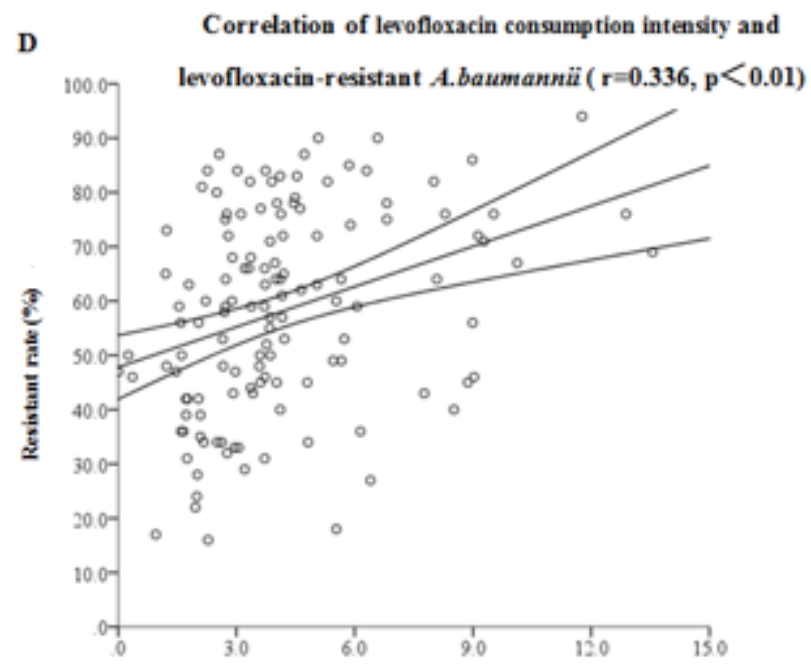

Levofloxacin consump tion intensity (DDDs per 100 patient-days)

\section{Figure 4}

Correlation of FQR A.baumannii and consumption intensity of (A) all antibiotics; (B) combinations of third-generation cephalosporins and beta-lactamase inhibitors; (C) fluoroquinolone; (D) levofloxacin. 3GC: the third generation cephalosporins; CBLI: combinations with beta-lactamase inhibitors. 